\title{
Substrate oxidation and the influence of breakfast in normobaric hypoxia and normoxia
}

\author{
Alex Griffiths $^{1}{ }^{1}$ $\cdot$ Kevin Deighton ${ }^{1} \cdot$ Oliver M. Shannon ${ }^{1,2} \cdot$ Jamie Matu ${ }^{1} \cdot$ Roderick King $^{1} \cdot$ John P. O'Hara ${ }^{1}$
}

Received: 2 April 2019 / Accepted: 17 June 2019 / Published online: 3 July 2019

(c) The Author(s) 2019

\begin{abstract}
Purpose Previous research has reported inconsistent effects of hypoxia on substrate oxidation, which may be due to differences in methodological design, such as pre-exercise nutritional status and exercise intensity. This study investigated the effect of breakfast consumption on substrate oxidation at varying exercise intensities in normobaric hypoxia compared with normoxia.

Methods Twelve participants rested and exercised once after breakfast consumption and once after omission in normobaric hypoxia (4300 m: $\mathrm{F}_{\mathrm{i}} \mathrm{O}_{2} \sim 11.7 \%$ ) and normoxia. Exercise consisted of walking for $20 \mathrm{~min}$ at $40 \%, 50 \%$ and $60 \%$ of altitudespecific $\mathrm{V}_{2 \max }$ at $10-15 \%$ gradient with a $10 \mathrm{~kg}$ backpack. Indirect calorimetry was used to calculate carbohydrate and fat oxidation.

Results The relative contribution of carbohydrate oxidation to energy expenditure was significantly reduced in hypoxia compared with normoxia during exercise after breakfast omission at $40 \%(22.4 \pm 17.5 \%$ vs. $38.5 \pm 15.5 \%, p=0.03)$ and $60 \% \dot{\mathrm{VO}}_{2 \max }(35.4 \pm 12.4$ vs. $50.1 \pm 17.6 \%, p=0.03)$, with a trend observed at $50 \% \dot{\mathrm{VO}}_{2 \max }(23.6 \pm 17.9 \%$ vs. $38.1 \pm 17.0 \%$, $p=0.07$ ). The relative contribution of carbohydrate oxidation to energy expenditure was not significantly different in hypoxia compared with normoxia during exercise after breakfast consumption at $40 \%(42.4 \pm 15.7 \%$ vs. $48.5 \pm 13.3 \%, p=0.99), 50 \%$ $(43.1 \pm 11.7 \%$ vs. $47.1 \pm 14.0 \%, p=0.99)$ and $60 \% \dot{\mathrm{VO}}_{2 \max }(54.6 \pm 17.8 \%$ vs. $55.1 \pm 15.0 \%, p=0.99)$.

Conclusions Relative carbohydrate oxidation was significantly reduced in hypoxia compared with normoxia during exercise after breakfast omission but not during exercise after breakfast consumption. This response remained consistent with increasing exercise intensities. These findings may explain some of the disparity in the literature.
\end{abstract}

Keywords Carbohydrate $\cdot$ Fat $\cdot$ Utilisation $\cdot$ Fasted $\cdot$ Fed $\cdot$ Altitude

Abbreviations

AUC Area under the curve

FFA Free fatty acids

$\mathrm{F}_{\mathrm{i}} \mathrm{O}_{2} \quad$ Fraction of inspired oxygen

HIF-1 $\alpha$ Hypoxia inducible factor 1 alpha
$\mathrm{P}_{\mathrm{i}} \mathrm{O}_{2} \quad$ Partial pressure of inspired oxygen

PPAR $\alpha$ Peroxisome proliferator-activated receptor alpha

RPE Rating of perceived exertion

SD Standard deviation

SE Standard error

$\dot{\mathrm{VCO}}_{2}$ Carbon dioxide production

Communicated by Susan Hopkins.

Alex Griffiths

A.Griffiths@leedsbeckett.ac.uk

Kevin Deighton

K.Deighton@leedsbeckett.ac.uk

Oliver M. Shannon

Oliver.Shannon@Newcastle.ac.uk

Jamie Matu

J.Matu@leedsbeckett.ac.uk

Roderick King

R.King@leedsbeckett.ac.uk
John P. O’Hara

J.OHara@leedsbeckett.ac.uk

1 Research Institute for Sport, Physical Activity and Leisure, Leeds Beckett University, Leeds LS6 3QS, UK

2 Human Nutrition Research Centre, Institute of Cellular Medicine, Newcastle University, Leech Building, Framlington Place, Newcastle Upon Tyne NE2 4HH, UK 
$\begin{array}{ll}\dot{\mathrm{V}} \mathrm{O}_{2} & \text { Oxygen uptake } \\ \dot{\mathrm{VO}}_{2 \max } & \text { Maximal oxygen update }\end{array}$

\section{Introduction}

Disparate metabolic responses have been observed during exercise matched for relative intensities in hypoxia compared with normoxia (Young et al. 1982; Braun et al. 2000; Beidleman et al. 2002; Lundby and Van Hall 2002; Friedmann et al. 2004; Péronnet et al. 2006; Katayama et al. 2010; Morishima et al. 2014; O'Hara et al. 2017; Matu et al. 2017). These contrasting findings within the literature appear to be due to differences in experimental design, specifically pre-exercise nutritional status and exercise intensity (Griffiths et al. 2019). Whilst the effect of pre-exercise breakfast consumption (Edinburgh et al. 2018) and exercise intensity (Van Loon et al. 2001) on metabolism are well documented in normoxic conditions, the metabolic response to these factors is yet to be quantified in hypoxia. In addition, due to the inconsistent use of pre-exercise breakfast consumption in the literature, a direct comparison of the two distinct states during exercise of varying intensities in normoxia and hypoxia may provide clarity on such equivocal findings. Further, whilst the use of studies utilising fasted participants to control for baseline metabolic status is warranted, knowledge of how this differs to fed participants is necessary to generate practical recommendations for relevant populations.

It has been proposed that during exercise matched for relative intensities, the relative contribution of carbohydrate oxidation to energy expenditure is higher in hypoxia compared with normoxia when performed after breakfast consumption, but lower in hypoxia than normoxia when exercise was performed after breakfast omission (Griffiths et al. 2019). A potential explanation of findings observed after breakfast consumption is that greater oxidation and mobilisation of endogenous carbohydrate stores may be stimulated via the combined effect of hypoxia (Katayama et al. 2010) and feeding (Tentolouris et al. 2003) on the sympathetic nervous system. Additionally, a similar effect of hypoxia (Matu et al. 2018) and feeding (Blom et al. 2005) may increase circulating insulin concentration and subsequently inhibit lipolysis and free fatty acid (FFA) mobilisation (Coyle et al. 1997). It also seems plausible that greater fat oxidation may be observed in hypoxia, compared with normoxia after breakfast omission. Increased expression of the transcription factor hypoxia inducible factor 1 alpha (HIF-1 $\alpha)$ may upregulate the fatty acid-activated transcription factor peroxisome proliferator-activated receptor alpha $(\operatorname{PPAR} \alpha)$ as per the metabolic response to hypoxia (Aragones et al. 2008). This response may be further stimulated by the fasted state (König et al. 1999), subsequently inhibiting pyruvate dehydrogenase activity (Huang et al. 2002) and enabling greater mobilisation and oxidation of fat stores (Spriet and Watt 2003).

Exercise intensity was also identified as a significant moderator of substrate oxidation during exercise matched to relative intensities in hypoxia (Griffiths et al. 2019). Specifically, the relative contribution of carbohydrate oxidation to energy expenditure was higher in hypoxia compared with normoxia during exercise performed at higher intensities. This was attributed to the hypoxic effect of both altitude and high intensity exercise, augmenting skeletal muscle hypoxia. The subsequent change in substrate oxidation could, therefore, be explained as per the normoxic response to increased exercise intensity (i.e., reduction in adipose tissue blood flow and lipolysis and/or downregulation of carnitine palmitoyltransferase-1) (Sahlin 1990; Romijn et al. 1993; Van Loon et al. 2001). Alternatively, sympathetic nervous system activity may be potentiated by hypoxia and greater exercise intensities, augmenting glycogenolysis and, therefore, carbohydrate oxidation (Watt et al. 2001).

An investigation into the effects of pre-exercise nutritional status and exercise intensity in hypoxia compared with normoxia may provide clarity on the current literature and facilitate the development of nutritional strategies for high altitude mountaineers and military personnel alike. As such, the purpose of this study was to investigate the effect of breakfast consumption or omission on substrate oxidation during exercise matched for relative intensities in normobaric hypoxia and normoxia. These exercise intensities ranged from $40-60 \%$ of altitude specific maximal oxygen uptake $\left(\dot{\mathrm{V}}_{2 \max }\right)$. We hypothesised that the relative contribution of carbohydrate oxidation to energy expenditure would be increased during exercise matched to relative intensities in hypoxia compared with normoxia after breakfast consumption, but that this response would not occur after breakfast omission. We also hypothesised that the relative contribution of carbohydrate oxidation would be potentiated with increasing exercise intensities in hypoxia compared with normoxia after both breakfast consumption and omission. Due to the differing metabolic response to hypoxia in females compared with males (Braun et al. 2000), all participants in the present study were male. Inclusion of a female sub-group was beyond the scope of the present study but warrants investigation in future studies.

\section{Methods}

\section{Participants}

Twelve, physically active (structured exercise $\geq 3$ times a week), healthy male volunteers ( $23 \pm 3$ years, $181.1 \pm 6.4 \mathrm{~cm}$, $79.8 \pm 13.1 \mathrm{~kg}$ ) provided written, informed consent to 
participate in this study. The study received institutional ethical approval (Leeds Beckett research ethics committee, application reference: 46180) and was conducted in accordance with the Declaration of Helsinki. All participants were non-smokers, normotensive, free from food allergies and were not taking any medication. None of the participants had travelled to an altitude of $>1500 \mathrm{~m}$ within the previous three months and were all currently residing at an altitude $<500 \mathrm{~m}$.

\section{Experimental design}

Participants were required to make a total of seven visits to the laboratory. The first visit involved pre-exercise screening, anthropometry, verbal familiarisation with testing procedures and a sickle cell trait test. Sickle cell trait was an exclusion criterion due to complications that may occur at altitude, for example, splenic infarction (Goodman et al. 2014). Further exclusion criteria included diabetes and thyroid disorders. The second and third visit required participants to be acutely exposed to normobaric hypoxia (fraction of inspired oxygen $\left(\mathrm{F}_{\mathrm{i}} \mathrm{O}_{2}\right)$ : 11.7\% when considering water vapour partial pressure (Conkin 2011; Fenn et al. 1946) and daily fluctuations in barometric pressure) equivalent to $4300 \mathrm{~m}$ (partial pressure of inspired oxygen $\left(\mathrm{P}_{\mathrm{i}} \mathrm{O}_{2}\right)$ : $83 \mathrm{mmHg}$ ) in an environmental chamber (TISS, Alton, UK and Sporting Edge, Sheffield on London, UK) or normoxia (absolute altitude $\sim 113 \mathrm{~m}$ ). Ambient temperature was maintained at $20{ }^{\circ} \mathrm{C}$ and relative humidity at $50 \%$ for all trials. Participants completed sub-maximal and maximal exercise tests to calculate walking speeds matched for relative exercise intensity in each environmental condition for the experimental trials. These two preliminary trials were separated by $\geq 48 \mathrm{~h}$ and conducted in a single-blind randomised fashion. On visits 4-7 the participants completed a 3-h 45 min experimental trial, which included a $2-\mathrm{h} 15 \mathrm{~min}$ rest period, followed by a $1-\mathrm{h}$ incremental walking protocol and a 30-min post-exercise rest period (Fig. 1). Two of the trials were performed in normobaric hypoxia equivalent to $4300 \mathrm{~m}$ [one trial with breakfast consumption (HB), and one with breakfast omission (HF)] and two were performed in normoxia [one trial with breakfast consumption (NB), and one with breakfast omission (NF)]. These visits were separated by $\geq 7$ days and were randomised independent of the preliminary trials, using a Latin Square design.

\section{Preliminary testing}

Participants completed an exercise test on a motorised treadmill (Woodway PPS S5; Waukesha, WI), which comprised of a sub-maximal and maximal phase. In the normoxic condition, the incremental sub-maximal phase involved four, 3-min stages walking at $3 \mathrm{~km} / \mathrm{h}, 4 \mathrm{~km} / \mathrm{h}, 4.5 \mathrm{~km} / \mathrm{h}$ and $5.5 \mathrm{~km} / \mathrm{h}$. Participants carried a $10 \mathrm{~kg}$ backpack to mimic the physiological demands of the experimental trials. The initial two walking speeds in the normoxic condition were performed at a $10 \%$ gradient and the second two at a $15 \%$ gradient. In normobaric hypoxia, participants walked at $1.5 \mathrm{~km} / \mathrm{h}, 2.5 \mathrm{~km} / \mathrm{h}, 3.5 \mathrm{~km} / \mathrm{h}$ and $4.5 \mathrm{~km} / \mathrm{h}$, at a $10 \%$ gradient throughout. Lower speeds and gradients were used in normobaric hypoxia based on the reduced $\mathrm{V}_{2 \max }$ elicited at altitude (Dill et al. 1931), and the need for all participants to achieve $60 \% \mathrm{~V}_{2 \max }$ within the 12 -min trial. The higher gradient utilised in normoxia was employed to ensure participants achieved $60 \% \mathrm{~V}_{2 \max }$ with a walking gait. Following completion of the sub-maximal phase, participants then rested for approximately $5 \mathrm{~min}$, after which the maximal phase of the test commenced. Within this $5 \mathrm{~min}$, participants

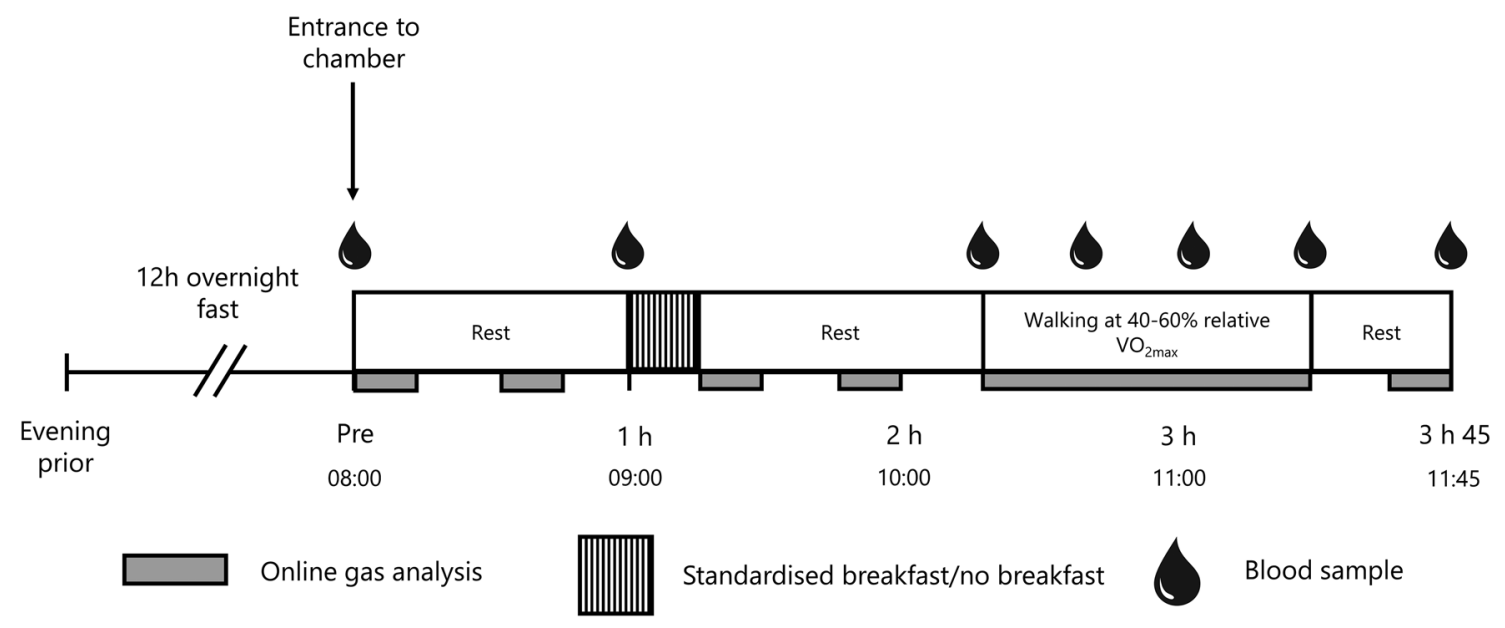

Fig. 1 Schematic of full experimental trial. Trials were completed twice in hypoxia (once with breakfast consumption, once with breakfast omission) and twice in normoxia (once with breakfast consump- tion, once with breakfast omission). Exercise involved $20 \mathrm{~min}$ at $40 \%$, $50 \%$ and $60 \%$ relative $\mathrm{VO}_{2 \max }$ 
were permitted to rest seated, or complete some light stretching. Participants ran without a backpack, at a $1 \%$ gradient (Jones and Doust 1996), at a constant speed dependant on fitness and environmental condition, aiming for a perceived exertion of 12 . The gradient was increased by $1 \%$ every minute until volitional exhaustion. Oxygen uptake $\left(\mathrm{V}_{2}\right)$ and carbon dioxide production $\left(\dot{\mathrm{VCO}}_{2}\right)$ measurements were made throughout both phases of the test using an online gas analysis system (Metalyser, Cortex, Germany), which was calibrated following the manufacturer's instructions. In this regard, the online gas analyser was calibrated with daily barometric pressure, a $3 \mathrm{~L}$ syringe (volume), as well as ambient gases and two known concentrations of gas (15\% $\mathrm{O}_{2}$ and $5 \% \mathrm{CO}_{2}$ ). These gases were subsequently checked before use with an acceptance limit set at $\pm 0.02 \%$ for both $\mathrm{O}_{2}$ and $\mathrm{CO}_{2}$. All participants were deemed to reach a 'true' $\dot{\mathrm{V}}{ }_{2 \text { max }}$ by fulfilment of $>2$ of the following criteria: a plateau in $\dot{\mathrm{VO}}_{2}$ in the final exercise stage, respiratory exchange ratio $\geq 1.15$, heart rate within $10 \mathrm{~b} \cdot \mathrm{min}^{-1}$ of age predicted maximum (220-age), rating of perceived exertion (RPE) $\geq 19$ and/or blood lactate $\geq 8 \mathrm{mM}$ (Howley et al. 1995). The sub-maximal and maximal data was used to establish walking speeds that would elicit $40 \%, 50 \%$ and $60 \% \dot{\mathrm{VO}}_{2 \max }$ relative to both normoxia and hypoxia whilst carrying a $10 \mathrm{~kg}$ backpack at a $10 \%$ gradient.

\section{Diet and physical activity before testing}

Participants recorded their food intake for the $24 \mathrm{~h}$ before the first experimental trial and were instructed to repeat the quantity and timing of this intake for each subsequent visit. During these $24 \mathrm{~h}$, participants were asked not to perform strenuous activity or consume caffeine or alcohol. Participant adherence to these requirements was verbally confirmed before each trial. In addition, on the day before each experimental trial, participants were provided with, and consumed a standardised evening meal at home between 7 and $8 \mathrm{pm}$ that included fusilli pasta, pasta sauce, cheddar cheese, milk and jelly beans (1037 kcal, 57\% carbohydrate, 28\% fat, 15\% protein). This meal was consumed to minimise the possibility of a second meal effect confounding glycaemic control or any other measured variables (Wolever et al. 1988; Stevenson et al. 2005).

\section{Experimental trials}

Participants arrived at the research facilities following a $12 \mathrm{~h}$ fast and entered the environmental chamber at $8 \mathrm{am}$. Verbal confirmation that participants had fasted for the previous $12 \mathrm{~h}$ was obtained prior to commencing each trial. Participants then rested for an hour. During rest periods, participants were seated upright and permitted to undertake personal activities such as reading. At $1 \mathrm{~h}$, in both the normobaric hypoxia and normoxia breakfast consumption trials, participants were allowed 15 min to consume a standardised breakfast (535 kcal, 58\% carbohydrate, 24\% fat, $18 \%$ protein). This meal included rolled oats, semi-skimmed milk and orange juice, and was designed to replicate typical breakfast consumption in the UK (Reeves et al. 2013). At $1 \mathrm{~h}$ in the normobaric hypoxia and normoxia breakfast omission trials, participants continued resting for $15 \mathrm{~min}$, without the consumption of breakfast. At $1 \mathrm{~h} 15 \mathrm{~min}$, participants in all trials rested for a further hour. At $2 \mathrm{~h} 15 \mathrm{~min}$, participants completed a 1 -h walking test (20 min at $40 \%$, $50 \%$ and $60 \% \dot{\mathrm{VO}}_{2 \max }$ ) at a $10 \%$ gradient, carrying a $10 \mathrm{~kg}$ backpack, to mimic the demands of high altitude trekking (Mellor et al. 2017). Participants then rested for 30 min after exercise. Water was allowed ad libitum throughout all trials. See Fig. 1 for a schematic representation of the experimental trials.

\section{Measurements}

\section{Heart rate, capillary oxygen saturation and RPE}

Heart rate and capillary oxygen saturation were measured using a fingertip pulse oximeter (Nellcor PM10N, United States) every 15 min during rest. Heart rate, capillary oxygen saturation and RPE were measured every 10-min throughout exercise.

\section{Expired breath analysis}

Expired gas breath samples were collected using an online gas analysis system (Metalyser, Cortex, Germany) for two 10 -min resting periods in the first hour (pre-prandial) of exposure (5-15 min and 35-45 $\mathrm{min}$ ). In the hour following breakfast consumption or omission (post-prandial), a further two 10-min resting periods of expired gas breath samples were collected ( $1 \mathrm{~h} 20 \mathrm{~min}-1 \mathrm{~h} 30 \mathrm{~min}$ and $1 \mathrm{~h} 50 \mathrm{~min}-2 \mathrm{~h}$ ). In addition, these measurements were made continuously throughout the 1-h walking protocol, and for the final $10 \mathrm{~min}$ in the 30-min post-exercise period. Participants were fitted with a facemask by researchers $5 \mathrm{~min}$ prior to the collection period whilst the participant was seated. At the end of the collection period, participants were asked to remove the facemask to minimise unnecessary opening and closing of the chamber door. This approach (5 min prior to collection and 10 min collection period) has demonstrated reliability previously (correlation coefficient: 0.8 ) (Spaeth et al. 2015). Coefficient of variation values from the pre-prandial period in the normoxia and normobaric hypoxia trials in the present study (repeated measurements under same conditions) were within 4-9\% as recommended by Skinner et al. (1999) (normoxia: $\dot{\mathrm{VO}}_{2}=6.2 \%, \dot{\mathrm{V} C O}=5.2 \%$; normobaric hypoxia: 
$\dot{\mathrm{VO}}_{2}=4.4 \%, \dot{\mathrm{VCO}}_{2}=5.2 \%$ ). Substrate oxidation was calculated using relevant equations for both resting (Frayn 1983) and exercise periods (Jeukendrup and Wallis 2005).

\section{Blood sampling}

Researchers entered the chamber to draw venous blood samples from a 20-gauge cannula (Introcan Safety; B Braun, Sheffield, UK) which was inserted into an antecubital vein upon arrival. Samples were drawn at baseline (before entry to the chamber) for the analysis of plasma glucose, plasma lactate, serum FFA and serum insulin. Subsequent blood samples variables were drawn at $1 \mathrm{~h}$ (pre-prandial), $2 \mathrm{~h} 15$ min (post-prandial), $2 \mathrm{~h} 35 \mathrm{~min}\left(40 \% \dot{\mathrm{V}}_{2 \max }\right), 2$ h $55 \min \left(50 \% \dot{\mathrm{VO}}_{2 \max }\right), 3 \mathrm{~h} 15 \mathrm{~min}\left(60 \% \dot{\mathrm{V}}_{2 \max }\right)$ and 3 h $45 \mathrm{~min}$ (post exercise) (no insulin at this time point due to plate layout). For samples in close proximity (i.e., during exercise), researchers stayed in the chamber to avoid unnecessary opening and closing of chamber doors. Fluoride oxalate tubes used for plasma glucose and lactate were spun at $1500 \times g$ for $10 \mathrm{~min}$ in a centrifuge (CompactStar CS4, VWR) immediately after being filled with venous blood. The serum separator tubes used for FFA and insulin were spun at the same speed, 30 min after collection to allow for clotting. The supernatant was then transferred into separate Eppendorf tubes to be frozen immediately at $-20{ }^{\circ} \mathrm{C}$ before being transferred to $-80{ }^{\circ} \mathrm{C}$ until analysis.

\section{Blood analysis}

Commercially available enzyme-linked immunosorbent assay kits were used to determine serum concentrations of insulin (IBL, Hamburg, Germany). To eliminate interassay variation, all samples from each participant were analysed on the same plate. Plasma glucose and lactate, and serum FFA were measured photometrically with reagents from Instrumentation Laboratories (Lexington, MA) and Randox Laboratories (Crumlin, UK). The within batch coefficients of variation were as follows: insulin $5.9 \%$, glucose $1.8 \%$, lactate $2.8 \%$ and FFA $3.7 \%$.

\section{Statistical analysis}

Data are expressed as mean \pm standard deviation (SD) in text and mean \pm standard error (SE) in figures. All data were analysed using IBM SPSS statistics (v24 for Windows; SPSS; Chicago, IL). The trapezoid method was used to calculate area under the curve (AUC) for substrate oxidation and hormone concentrations. The periods of AUC were defined as pre-prandial ( $0-1 \mathrm{~h})$, post-prandial ( $1 \mathrm{~h} 15 \mathrm{~min}-2 \mathrm{~h} 15 \mathrm{~min})$, $40 \%$ relative $\dot{\mathrm{VO}}_{2 \max }(2 \mathrm{~h} 15 \mathrm{~min}-2 \mathrm{~h} 35 \mathrm{~min}$ ), $50 \%$ relative $\dot{\mathrm{VO}}_{2 \text { max }}(2 \mathrm{~h} 35 \mathrm{~min}-2 \mathrm{~h} 55 \mathrm{~min}), 60 \%$ relative $\dot{\mathrm{VO}}_{2 \text { max }}(2$ h 55 min-3 h 15 min) and post exercise ( $3 \mathrm{~h} 15$ min -3 h $45 \mathrm{~min}$ ). Normality of distribution was evaluated using histograms and Shapiro-Wilk test and approximated normal distribution. A paired sample $t$ test was used to determine differences between $\dot{\mathrm{VO}}_{2 \max }$ in normoxic and hypoxic conditions. One-way repeated measures ANOVA was used to determine differences between trials for energy expenditure, heart rate, capillary oxygen saturation and RPE. Two-way repeated measures ANOVA (time $\times$ trial) was used to determine differences between absolute and relative carbohydrate and fat oxidation and hormone concentrations between AUC periods. Where significant main effects of trial were found, further post hoc analysis was performed using Bonferroni correction for multiple comparisons. Effect sizes are presented as Cohen's $d$ and interpreted as $\leq 0.2$ trivial, $>0.2$ small, $>0.6$ moderate, $>1.2$ large, $>$ very large and $>4$ extremely large (Hopkins 2004).

\section{Results}

\section{Maximal oxygen uptake and walking speeds}

$\dot{\mathrm{V}} \mathrm{O}_{2 \max }$ was significantly reduced in hypoxia compared with normoxia $\left(38.3 \pm 6.0 \mathrm{~mL} \cdot \mathrm{kg}^{-1} \cdot \mathrm{min}^{-1} \mathrm{vs}\right.$. $\left.53.0 \pm 8.6 \mathrm{~mL} \cdot \mathrm{kg}^{-1} \cdot \mathrm{min}^{-1} ; p<0.001, d=2.00\right)$. In hypoxia, this elicited walking speeds of $1.8 \pm 0.4 \mathrm{~km} \cdot \mathrm{h}^{-1}$ (HB: $40.3 \pm 4.1 \%$; HF: $\left.38.9 \pm 3.1 \% \dot{\mathrm{VO}}_{2 \max }\right), 2.7 \pm 0.5 \mathrm{~km} \cdot \mathrm{h}^{-1}$ (HB: $47.8 \pm 3.3 \% \dot{\mathrm{VO}}_{2 \max }$; HF: $48.0 \pm 4.4 \% \dot{\mathrm{VO}}_{2 \max }$;) and $3.5 \mathrm{~km} \cdot \mathrm{h}^{-1}$ (HB: $59.6 \pm 5.9 \% \dot{\mathrm{VO}}_{2 \max }$; HF: $59.1 \pm 5.0 \%$ $\left.\dot{\mathrm{V}}_{2 \max }\right)$. In normoxia, this elicited walking speeds of $3.4 \pm 0.3 \mathrm{~km} \cdot \mathrm{h}^{-1}$ (NB: $38.4 \pm 3.4 \% \dot{\mathrm{VO}}_{2 \max } ; \mathrm{NF}: 38.1 \pm 3.9 \%$ $\dot{\mathrm{VO}}_{2 \max }$ ), $4.1 \pm 0.4 \mathrm{~km} \cdot \mathrm{h}^{-1}$ (NB: $45.8 \pm 3.3 \% \dot{\mathrm{VO}}_{2 \max } ; \mathrm{NF}$ : $\left.45.1 \pm 2.9 \% \dot{\mathrm{VO}}_{2 \max }\right)$ and $4.6 \pm 0.5 \mathrm{~km} \cdot \mathrm{h}^{-1}(\mathrm{NB}: 61.4 \pm 2.5 \%$ $\dot{\mathrm{V}} \mathrm{O}_{2 \max } ; \mathrm{NF}: 61.3 \pm 3.3 \% \dot{\mathrm{VO}}_{2 \max }$ ) for each 20 -min exercise period. Relative exercise intensity was not significantly different between any trial at $40 \%(p=0.39)$, or $60 \% \dot{\mathrm{VO}}_{2 \max }$ $(p=0.18)$, however, a trend for an increased relative exercise intensity in hypoxia compared with normoxia after breakfast omission was observed at $50 \% \dot{\mathrm{VO}}_{2 \max }(p=0.06)$.

\section{Experimental trials}

\section{Energy expenditure}

Energy expenditure at rest was significantly greater in hypoxia compared with normoxia in both the breakfast consumption $(1252 \pm 158 \mathrm{~kJ}$ vs. $1108 \pm 145 \mathrm{~kJ} ; p=0.02$, $d=0.95)$ and breakfast omission trials $(1349 \pm 250 \mathrm{~kJ}$ vs. $1053 \pm 140 \mathrm{~kJ} ; p=0.001, d=1.52)$. Energy expenditure at rest was not significantly different between breakfast consumption and omission in hypoxia ( $p=0.66, d=0.38)$ or normoxia $(p=0.49, d=0.47)$. 
Energy expenditure during exercise was significantly reduced in hypoxia compared with normoxia after both breakfast consumption $(1809 \pm 218 \mathrm{~kJ}$ vs. $2477 \pm 205 \mathrm{~kJ}$ $p<0.001, d=3.16)$ and omission $(1734 \pm 223 \mathrm{~kJ}$ vs. $2425 \pm 262 \mathrm{~kJ}, p<0.001, d=2.83)$. Energy expenditure during exercise was not significantly different between breakfast consumption and omission in hypoxia $(p=0.34, d=0.22)$ and normoxia $(p=0.99, d=0.33)$.

\section{Pre-prandial carbohydrate and fat oxidation}

In the pre-prandial period, absolute (Table 1) carbohydrate oxidation $(p \geq 0.11, d \leq 0.86)$ and its relative contribution to energy expenditure were not significantly different between trials (HB: $43.8 \pm 16.8 \%$, HF: $40.8 \pm 24.0 \%$, NB: $34.8 \pm 16.2 \%$, NF: $38.4 \pm 15.5 \%, p \geq 0.63, d \leq 0.54)$. In the same period, absolute $(p=0.99, d \leq 0.42$, Table 1$)$ and relative contributions of fat oxidation were not significantly different between trials (HB: $56.2 \pm 16.8 \%$, HF: $59.2 \pm 24.0 \%$, NB: $65.2 \pm 16.2 \%$, NF: $61.6 \pm 15.5 \%, p \geq 0.63, d \leq 0.54)$.

\section{Post-prandial carbohydrate and fat oxidation}

In the post-prandial period, absolute carbohydrate oxidation (Table 1) was significantly higher after breakfast consumption, compared with breakfast omission in hypoxia (absolute: $p<0.001, d=1.92)$ and normoxia $(p=0.04, d=1.25)$. In addition, the relative contribution of carbohydrate oxidation was significantly higher after breakfast consumption, compared with breakfast omission in hypoxia $(46.2 \pm 14.1 \%$ vs. $19.6 \pm 14.9 \%, p<0.001 ; d=1.84)$, but not normoxia $(45.5 \pm 15.4 \%$ vs. $29.6 \pm 16.9 \%, p=0.14, d=0.99)$. Absolute carbohydrate oxidation was not significantly different between hypoxia and normoxia after breakfast consumption $(p=0.50, d=0.56)$ or omission $(p=0.99, d=0.20)$. The relative contribution of carbohydrate oxidation was not significantly different between hypoxia and normoxia after breakfast consumption ( $p=0.99, d=0.05$ ) or omission $(p=0.56, d=0.63)$.

In the same period, absolute fat oxidation was significantly higher after breakfast omission, compared with consumption in hypoxia $(p<0.01, d=1.72)$ but not normoxia $(p=0.99, d=0.49)$. In addition, the relative contribution of fat oxidation was significantly higher after breakfast omission, compared with consumption in hypoxia $(80.4 \pm 14.9$ vs. $53.8 \pm 14.1, p<0.001, d=1.84)$ but not normoxia ( $70.4 \pm 16.9$ vs. $54.5 \pm 15.4, p=0.14, d=0.99)$. Absolute fat oxidation was significantly higher in hypoxia compared with normoxia after breakfast omission $(p<0.01, d=1.72)$ but not consumption ( $p=0.48, d=0.68$ ). The relative contribution of fat oxidation was not significantly different between hypoxia and normoxia after breakfast omission $(p=0.56$, $d=0.63$ ), or consumption ( $p=0.99, d=0.05$ ).

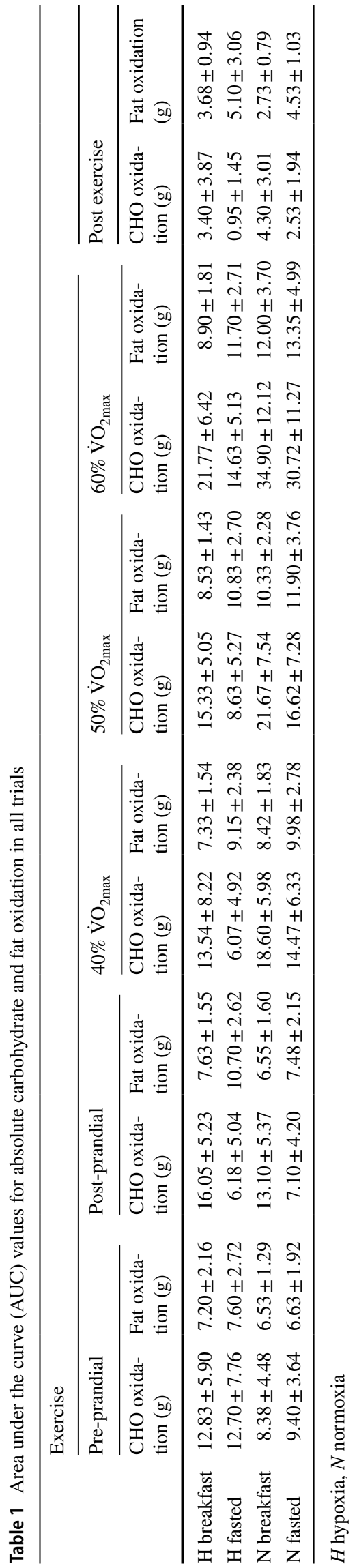




\section{Exercise carbohydrate and fat oxidation}

During exercise at all intensities, absolute (Table 1) carbohydrate oxidation was significantly lower in hypoxia compared with normoxia after breakfast omission $(40 \%$ $p<0.01, d=1.49 ; 50 \% p=0.01, d=1.27 ; 60 \% p=0.001$, $d=1.96)$. Absolute carbohydrate oxidation was also significantly lower in hypoxia compared with normoxia after breakfast consumption at $60 \% \dot{\mathrm{VO}}_{2 \max }(p=0.02, d=1.42)$, and approached significance at $50 \% \dot{\mathrm{V}}_{2 \max }(p=0.06$, $d=1.00)$, but not at $40 \% \dot{\mathrm{VO}}_{2 \max }(p=0.71, d=0.71)$. The relative contribution of carbohydrate (with the exclusion of $50 \% \dot{\mathrm{V}}_{2 \max }$ ) was significantly lower in hypoxia, compared with normoxia after breakfast omission $(40 \% p=0.03$; $d=0.98 ; 50 \% p=0.07, d=0.83 ; 60 \% p=0.03, d=0.98$ ) but not breakfast consumption at any intensity (all $p=0.99$, $d \leq 0.42$ ). Absolute carbohydrate oxidation was significantly higher after breakfast consumption compared with omission at all intensities in hypoxia ( $40 \%: p=0.02, d=1.14$; $50 \%: p=0.001, d=1.30 ; 60 \%: p<0.01, d=1.24)$ but not normoxia $(p \geq 0.09, d=0.68)$. The relative contribution of carbohydrate oxidation was significantly higher after breakfast consumption, compared with omission at all intensities in hypoxia (40\%: $p<0.01, d=1.20 ; 50 \%: p<0.01, d=1.32$; $60 \% p=0.01, d=1.28$ ), but not in normoxia (all $p \geq 0.28$, $d \leq 0.69$ ).

During exercise, absolute fat oxidation (Table 1) was not significantly different between hypoxia and normoxia at any exercise intensity after breakfast consumption $(p \geq 0.14$, $d \leq 1.12$ ) or omission (all $p=0.99, d \leq 0.43$ ). The relative contribution of fat oxidation (with the exclusion of $50 \% \dot{V}$ $\mathrm{O}_{2 \max }$ ) (Fig. 2) was significantly higher in hypoxia compared with normoxia after breakfast omission $(40 \%: p=0.03$, $d=0.98 ; 50 \%: p=0.07 ; d=0.83 ; 60 \%: p=0.03, d=0.98$ ) but not at any intensity after breakfast consumption (all $p=0.99, d \leq 0.42$ ). In addition, absolute fat oxidation was significantly higher at all exercise intensities after breakfast omission compared with consumption in hypoxia (40\%: $p=0.04, d=0.93 ; 50 \%: p=0.02, d=1.12 ; 60 \%: p=0.02$, $d=1.24)$ but not normoxia ( $p \geq 0.58, d \leq 0.68)$. The relative contribution of fat oxidation was significantly higher at all exercise intensities after breakfast omission compared with consumption in hypoxia ( $40 \%$ : $p<0.01, d=1.20 ; 50 \%$ : $p<0.01, d=1.32 ; 60 \%$ : $p=0.01, d=1.28)$, but not normoxia $(p \geq 0.28, d \leq 0.69)$.

\section{Post-exercise carbohydrate and fat oxidation}

In the post-exercise period, absolute carbohydrate oxidation was not significantly different between trials $(p \geq 0.12$, $d \leq 0.92$ ). The relative contribution of carbohydrate oxidation was also not significant between trials (HB: $19.9 \pm 19.8 \%$, HF: $6.6 \pm 10.0 \%$, NB: $30.01 \pm 21.2 \%$, NF: $19.7 \pm 15.5 \%$,
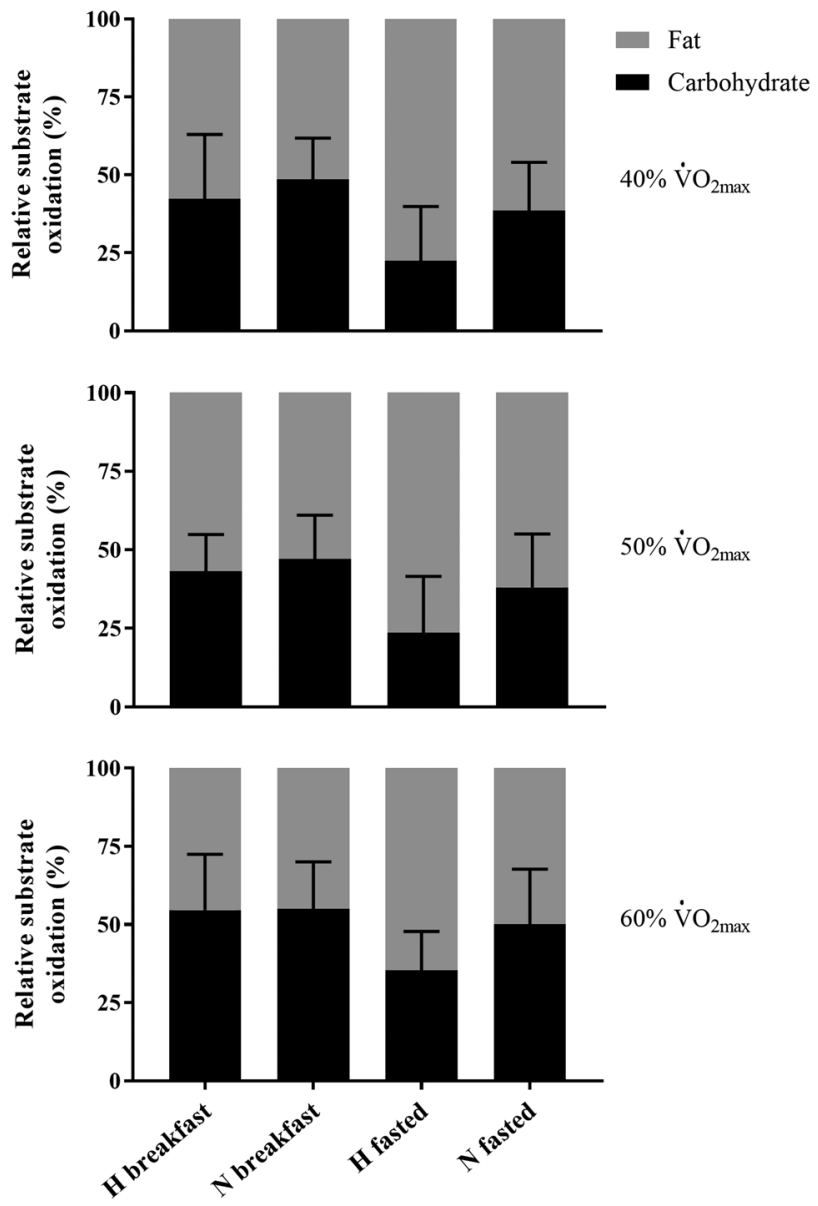

Fig. 2 The relative (\% energy yield) contribution of carbohydrate and fat oxidation during uphill walking at $40 \%, 50 \%$ and $60 \% \dot{\mathrm{VO}}_{2 \max }$ in normoxia and hypoxia after breakfast consumption and omission

$p \geq 0.10, d \leq 1.02)$. In the same period, absolute fat oxidation was significantly higher after breakfast omission compared with consumption in normoxia ( $p=0.001, d=1.97)$, but not hypoxia $(p=0.85, d=0.71)$. Absolute fat oxidation was not significantly different between hypoxia and normoxia after breakfast consumption ( $p=0.10, d=1.10)$ or omission $(p=0.99, d=0.28)$. The relative contribution of fat oxidation was not significantly different between any trial (HB: $80.1 \pm 19.8 \%$, HF: $93.4 \pm 10.0 \%$, NB: $69.9 \pm 21.2 \%$, NF: $80.3 \pm 15.5 \%, p \geq 0.10, d \leq 1.02)$.

\section{Blood biochemistry}

A significant effect of time (all $p<0.01$ ) and trial (all $p<0.01$ ) was observed for all analytes. Further, a significant interaction effect of time $\mathrm{x}$ trial was also observed for all analytes (all $p \leq 0.03$ ). All significant pairwise statistical comparisons are presented in Fig. 3. 


\section{Heart rate, capillary oxygen saturation and RPE}

Capillary oxygen saturation, heart rate, and RPE scores for the duration of the experimental trial are presented in Table 2. There were no significant differences between trials for heart rate $(p \geq 0.14, d \leq 0.96)$ and RPE $(p \geq 0.86$, $d \leq 0.21$ ). Capillary oxygen saturation was significantly lower in hypoxia compared with normoxia in both the breakfast consumption and omission trials $(p<0.01, \mathrm{~d}=7.83)$ $(p<0.01, d=9.63)$.

\section{Discussion}

This study investigated the effect of breakfast consumption and exercise intensity on substrate oxidation in hypoxia compared with normoxia during both rest and exercise. In this regard, the relative carbohydrate contributions to energy expenditure decrease, while relative fat contributions increase, during exercise matched for relative intensities in hypoxia compared with normoxia after breakfast omission, but not consumption. This effect of breakfast consumption in hypoxia compared with normoxia appears to be exclusive to exercise, with no differences in relative substrate oxidation between hypoxia and normoxia after breakfast consumption
Table 2 Mean capillary oxygen saturation $\left(\mathrm{SpO}_{2}\right)$, heart rate and RPE in all trials

\begin{tabular}{llll}
\hline & $\mathrm{SpO} 2$ & Heart rate & RPE \\
\hline H breakfast & $79 \pm 3$ & $86 \pm 9$ & $12 \pm 2$ \\
H fasted & $80 \pm 4$ & $88 \pm 21$ & $12 \pm 2$ \\
N breakfast & $79 \pm 3$ & $86 \pm 9$ & $12 \pm 2$ \\
$\mathrm{~N}$ fasted & $97 \pm 1^{*}$ & $75 \pm 13$ & $12 \pm 2$ \\
\hline
\end{tabular}

$H$ hypoxia, $N$ normoxia

*Denotes significance in comparison with corresponding nutritional status in hypoxia

or omission at rest. Higher exercise intensities did not potentiate carbohydrate oxidation in hypoxia, compared with normoxia after either breakfast consumption or omission.

Absolute carbohydrate oxidation was significantly lower in hypoxia compared with normoxia during exercise at all intensities after breakfast omission. It has long been established that hypoxia induces a lower $\mathrm{V}_{2 \max }$ than normoxia (Dill et al. 1931), which subsequently elicits lower absolute workloads during exercise matched to relative intensities in hypoxia, compared with normoxia (Lundby and Van Hall 2002). As such, the reduction in absolute carbohydrate oxidation in hypoxia, compared with normoxia after breakfast
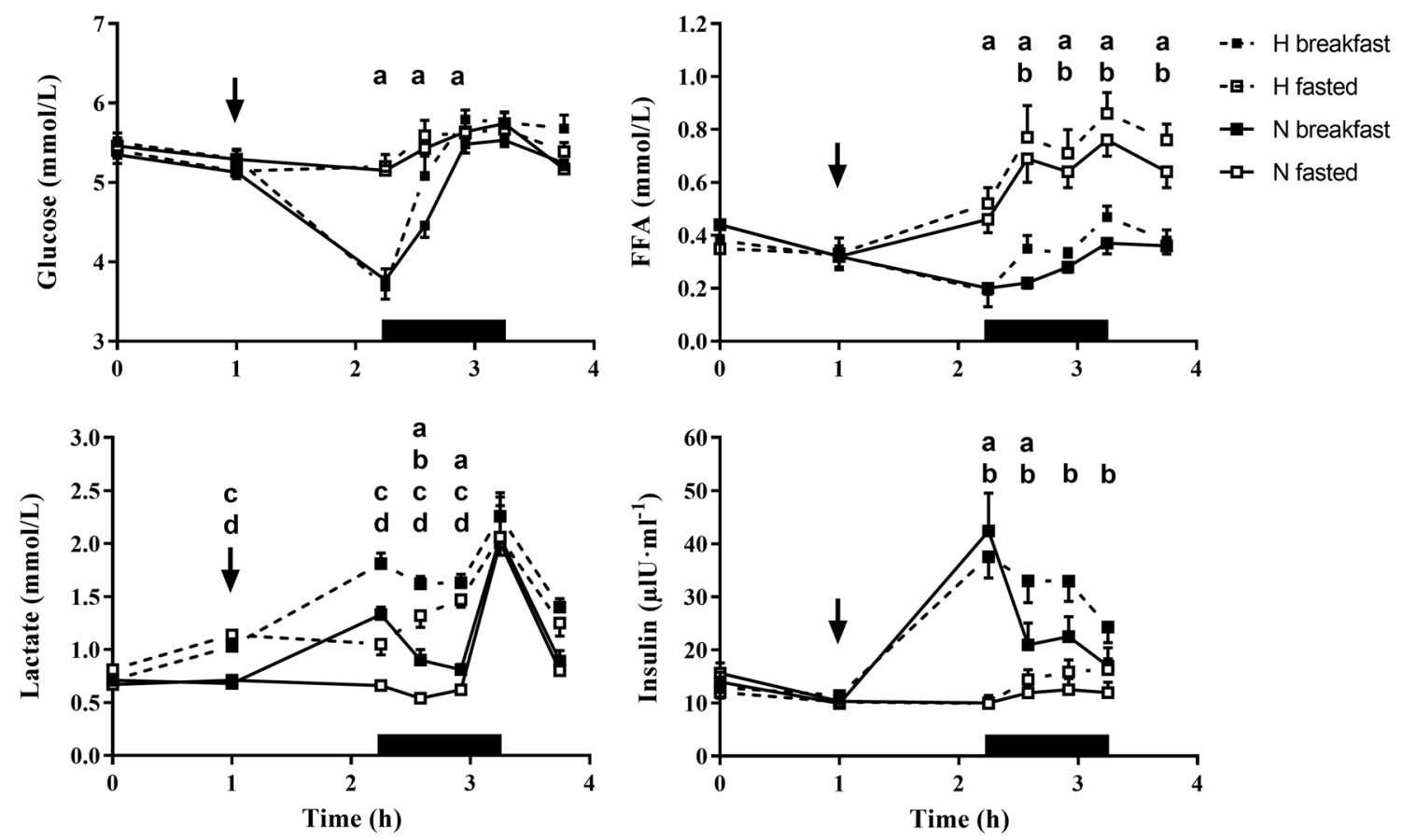

Fig. 3 Plasma glucose, plasma lactate, serum FFA and serum insulin concentrations over the full experimental trial. Values are mean \pm SE. The thin arrow represents the timing of breakfast in the hypoxic and normoxic breakfast consumption trials. Breakfast was not consumed in the hypoxic and normoxic fasted trials. The black rectangle represents the exercise period. a A significant difference between breakfast

consumption and omission in normoxia. b A significant difference between breakfast consumption and omission in hypoxia. c A significant difference between hypoxia and normoxia after breakfast consumption. d A significant difference between hypoxia and normoxia after breakfast omission. Significance $p<0.05$ 
omission is likely due in part to the reduced energy expenditure during exercise in hypoxia. Interestingly, this effect was less pronounced during exercise after breakfast consumption in hypoxia compared with normoxia (significance at $60 \%$ $\dot{\mathrm{VO}}_{2 \max }$ only and approaching significance at $50 \% \dot{\mathrm{VO}}_{2 \max }$ ). However, due to the confounding factor associated with utilising absolute substrate oxidation, the use of substrate oxidation data relative to total energy expenditure is warranted to determine an effect of hypoxia beyond a reduced absolute workload.

A novel finding of this study is the lower relative contribution of carbohydrate and higher relative contribution of fat oxidation observed during exercise matched for relative intensities in hypoxia, compared with normoxia after breakfast omission. This finding is in agreement with some existing literature utilising overnight fasted male participants in acute hypoxia (O'Hara et al. 2017) but not others (Katayama et al. 2010; Morishima et al. 2014). O'Hara et al. (2017) observed an increased relative contribution of fat to energy expenditure during exercise matched to relative intensities $\left(74 \% \dot{\mathrm{VO}}_{2 \max }\right)$ in hypoxia compared with normoxia. It was proposed that this may in part, be associated with augmented lipolysis and, therefore, FFA oxidation. The increased rates of lipolysis were supported by elevated concentrations of metanephrine and normetanephrine, as well as a subsequent increase in circulating FFA. This effect was observed despite consumption of a carbohydrate drink in both conditions (1.2 $\mathrm{g} \cdot \mathrm{min}^{-1}$ glucose, $0.6 \mathrm{~g} \cdot \mathrm{min}^{-1}$ fructose), demonstrating the potency of being fasted during exercise in hypoxia. In the present study, no significant differences in FFA concentrations were observed between hypoxia and normoxia after breakfast consumption or omission. This suggests that the higher relative contribution of fat oxidation observed in hypoxia, compared with normoxia after breakfast omission in the present study may not be associated with increased lipolysis of triglycerides stored in adipose tissue. As such, it seems plausible that an increased oxidation of intramuscular trigylcerides which would not influence circulating FFA concentrations, may contribute to the increased relative contribution of fat oxidation during exercise after breakfast omission in hypoxia, compared with normoxia.

At the mitochondrial level, it has been proposed that the increased relative contribution of fat oxidation to energy expenditure in hypoxia may be a result of increased expression of the transcription factor HIF- $1 \alpha$ and the upregulation of PPAR $\alpha$ (Aragones et al. 2008). Specifically, PPAR $\alpha$ has been demonstrated to deactivate pyruvate dehydrogenase (Huang et al. 2002), inhibiting the conversion of pyruvate to acetyl-coA and, therefore, enabling greater mobilisation and oxidation of fat stores (Spriet and Watt 2003). Subsequently, pyruvate may then be shunted towards lactate production and away from oxidative metabolism. Logically, the reduced relative carbohydrate oxidation observed after breakfast omission in hypoxia, compared with normoxia may be associated with a potentiated PPAR $\alpha$ response induced by fasting (König et al. 1999). Therefore, as expected, increased lactate concentrations were observed after breakfast omission in hypoxia, compared with normoxia however, this effect was also evident after breakfast consumption. Albeit, lactate concentrations in the fed state may also be inflated by the metabolism of fructose, derived from the consumption of orange juice during breakfast. Specifically, fructose metabolism can occur without the rate limiting step of glycolysis (catalysed by phosphofructokinase) and is, therefore, rapidly phosphorylated leading to increased rates of glycolysis and elevated plasma lactate concentrations (Jentjens et al. 2004; Tappy 2018).

In contrast to findings from our recent meta-analysis (Griffiths et al. 2019), we observed no significant change in the relative contribution of carbohydrate or fat to energy expenditure during exercise matched for relative intensities in hypoxia, compared with normoxia after breakfast consumption. This finding is in accordance with a number of studies investigating substrate oxidation during exercise matched to relative intensities in hypoxia, compared with normoxia (Lundby and Van Hall 2002; Young et al. 1982). However, it is in contrast to those who observed an increased relative contribution of carbohydrate (Péronnet et al. 2006; Friedmann et al. 2004) and fat (Matu et al. 2017; Braun et al. 2000) to energy expenditure in the same conditions. The variance in the literature regarding the use of fed participants is difficult to explain, but may be due to the numerous differing experimental characteristics such as carbohydrate supplementation and the sex of participants. The isolation of each of these characteristics in randomised control trials is required to further understand their influence on substrate oxidation in hypoxia.

Absolute carbohydrate oxidation was significantly higher in the post-prandial period after breakfast consumption compared with omission in both hypoxia and normoxia. Interestingly, the increased absolute carbohydrate oxidation observed after breakfast consumption in hypoxia and normoxia was associated with lower plasma glucose concentrations than breakfast omission. This effect remained significant at $40 \%$ and $50 \% \mathrm{VO}_{2 \max }$ in normoxia and approached significance at $40 \% \dot{\mathrm{VO}}_{2 \max }$ in hypoxia. This is likely due to the synergistic effect of augmented insulin concentrations and skeletal muscle contraction during exercise on GLUT-4 trafficking, subsequently inducing an alteration from fat to carbohydrate metabolism (Geiger et al. 2006). Evidence from Edinburgh et al. (2018) suggests that the upregulation of carbohydrate metabolism induced by insulin secretion in the post-prandial state is not solely matched by the subsequent glucose delivery to the muscle, and that for a period before feeding-derived carbohydrate entering the blood stream, muscle glycogen stores are also utilised. Taylor 
et al. (1993) also observed a reduction in muscle glycogen concentrations $1 \mathrm{~h}$ post-meal, before increasing again in the subsequent hours (1-7 h). Whilst we cannot confirm this in the present study, this may have implications for the timing of breakfast before exercise, and also provide a consideration for an additional exogenous carbohydrate source during exercise if the primary goal is to spare muscle glycogen and improve performance.

Alterations in substrate oxidation between hypoxia and normoxia after both breakfast consumption and omission were consistent across varying exercise intensities, suggesting that increasing exercise intensities may not potentiate carbohydrate oxidation in hypoxia, as previously proposed (Griffiths et al. 2019). However, the range of exercise intensities used in the present study were low to moderate intensity (40-60\% $\dot{\mathrm{VO}}_{2 \max }$ ) and, therefore, may not have been sufficient to potentiate sympathetic nervous system activity and glycogenolysis, as plasma epinephrine concentrations have been demonstrated to increase exponentially with exercise intensities increasing up to $85 \% \dot{\mathrm{VO}}_{2 \max }$ (Romijn et al. 1993).

The finding that energy expenditure at rest was significantly higher in normobaric hypoxia compared with normoxia is consistent with numerous other studies (Butterfield et al. 1992; Matu et al. 2017). An increase in resting energy expenditure and/or a reduction in energy intake has been associated with a negative energy balance and, therefore, weight loss during chronic hypoxic exposure (Armellini et al. 1997; Sergi et al. 2010). The increase in resting energy expenditure has been attributed to the elevated cardiovascular and ventilatory responses experienced during hypoxic exposure (Butterfield 1999). The reduction in energy intake observed in hypoxia is likely explained by impaired appetite regulation, a result of the hypoxic-induced suppression of the orexigenic hormone, acylated ghrelin (Debevec 2017; Matu et al. 2017). Whilst a reduction in body mass may induce debilitating effects on performance for high altitude mountaineers and military personnel alike, this finding may have implications for weight loss strategies in obese populations (Kayser and Verges 2013). The finding that energy expenditure during exercise was reduced in normobaric hypoxia compared with normoxia substantiates previous research (Matu et al. 2017), and is likely due to the reduced absolute workload in hypoxia. However, this reduced workload in hypoxia has been suggested to induce a similar metabolic load to normoxia, therefore, facilitating physical activity adherence while reducing the risk of musculoskeletal injury in obese individuals (Girard et al. 2017).

Despite the novel findings of this study, some notable limitations must be acknowledged. The use of muscle biopsies to investigate a key theory involving PPAR $\alpha$ was beyond the scope of this study and as such, a physiological explanation for the changes in substrate oxidation in the fasted state could not be confirmed. Further, whilst we could calculate whole body substrate oxidation, muscle/liver glycogen oxidation could not be analysed without muscle biopsies or tracer derived methods. In addition, the present study only investigated changes in young men; therefore, caution should be applied when applying the results to other populations. For example, women have been shown to elicit differing metabolic responses to hypoxia, when compared with men (Braun et al. 2000). Finally, the use of a normobaric hypoxic chamber in the present study should be acknowledged when considering the practical applicability of these findings to terrestrial altitude. Future research should investigate the effect of nutritional strategies (i.e., carbohydrate supplementation) in hypoxia after both breakfast consumption and omission to determine their effect on substrate oxidation and performance. In addition, it would be necessary to investigate substrate oxidation during exercise across a broader range of exercise intensities to determine if high intensity exercise induces different substrate oxidation responses than low intensity exercise when performed in hypoxia compared with normoxia.

\section{Conclusions}

In conclusion, we observed a reduced relative contribution of carbohydrate oxidation to energy expenditure during exercise matched for relative intensities in hypoxia, compared with normoxia after breakfast omission but no difference was observed during exercise after breakfast consumption. The effect of hypoxia on substrate oxidation was not altered with increasing exercise intensities when compared with normoxia. These data provide clarity on the current literature and may be useful in the design of nutritional strategies for high altitude mountaineers and military personnel.

Acknowledgements Funding for the study was provided internally by Leeds Beckett University. The authors would like to thank all participants for their time and commitment in undertaking this study.

Author Contribution AG, JOH, KD and RK conceived and designed the study. AG, OS and JM collected the data. AG analysed the data and wrote the manuscript. All authors read and provided critical feedback on the manuscript before approving.

\section{Compliance with ethical standards}

Conflict of interest The authors declare that they have no conflict of interest.

Ethical approval All procedures performed in studies involving human participants were in accordance with the ethical standards of Leeds Beckett University School of Sport research ethics committee and with the 1964 Helsinki declaration and its later amendments or comparable ethical standards. 
Informed consent Informed consent was obtained from all individual participants included in the study.

Open Access This article is distributed under the terms of the Creative Commons Attribution 4.0 International License (http://creativeco mmons.org/licenses/by/4.0/), which permits unrestricted use, distribution, and reproduction in any medium, provided you give appropriate credit to the original author(s) and the source, provide a link to the Creative Commons license, and indicate if changes were made.

\section{References}

Aragones J, Schneider M, Van Geyte K, Fraisl P, Dresselaers T, Mazzone M, Dirkx R, Zacchigna S, Lemieux H, Jeoung NH, Lambrechts D, Bishop T, Lafuste P, Diez-Juan A, Harten SK, Van Noten P, De Bock K, Willam C, Tjwa M, Grosfeld A, Navet R, Moons L, Vandendriessche T, Deroose C, Wijeyekoon B, Nuyts J, Jordan B, Silasi-Mansat R, Lupu F, Dewerchin M, Pugh C, Salmon P, Mortelmans L, Gallez B, Gorus F, Buyse J, Sluse F, Harris RA, Gnaiger E, Hespel P, Van Hecke P, Schuit F, Van Veldhoven P, Ratcliffe P, Baes M, Maxwell P, Carmeliet P (2008) Deficiency or inhibition of oxygen sensor Phd1 induces hypoxia tolerance by reprogramming basal metabolism. Nat Genet 40(2):170-180. https ://doi.org/10.1038/ng.2007.62

Armellini F, Zamboni M, Robbi R, Todesco T, Bissoli L, Mino A, Angelini G, Micciolo R, Bosello O (1997) The effects of high altitude trekking on body composition and resting metabolic rate. Horm Metab Res 29(9):458-461

Beidleman BA, Rock PB, Muza SR, Fulco CS, Gibson LL, Kamimori GH, Cymerman A (2002) Substrate oxidation is altered in women during exercise upon acute altitude exposure. Med Sci Sports Exerc 34(3):430-437

Blom W, Stafleu A, de Graaf C, Kok F, Schaafsma G, Hendriks H (2005) Ghrelin response to carbohydrate-enriched breakfast is related to insulin. Am J Clin Nutr 81:367-375

Braun B, Mawson J, Muza SR, Dominick S, Brookes G, Horning M, Rock PB, Moore L, Mazzeo R, Ezeji-Okoye S, Butterfield G (2000) Women at altitude Carbohydrate utilisation during exercise at 4300m. J Appl Physiol 88(1):246-256

Butterfield GE (1999) Nutrient requirements at high altitude. Clin Sports Med 18(3):607

Butterfield GE, Gates J, Fleming S, Brooks GA, Sutton JR, Reeves JT (1992) Increased energy intake minimises weight loss in men at high altitude. J Appl Physiol 72:1741-1748

Conkin J (2011) PH2O and simulated hypobaric hypoxia. Aviat Space Environ Med 82:1157-1158

Coyle E, Jeukendrup A, Wagenmakers A, Saris W (1997) Fatty acid oxidation is directly regulated by carbohydrate metabolism during exercise. Am J Physiol 273:E268-E275

Debevec T (2017) Hypoxia-related hormonal appetite modulation in humans during rest and exercise: mini review. Front Physiol 30(8):366

Dill DB, Edwards HT, Folling A, Oberg AM, Pappenheimer AM, Talbott JH (1931) Adaptations of the organism to changes in oxygen pressure. J Physiol 71

Edinburgh RM, Hengist A, Smith HA, Travers RL, Koumanov F, Betts JA, Thompson D, Wahlin JP, Hamilton DL, Stevenson EJ, Tipton KD, Gonzalez JT (2018) Pre-exercise breakfast ingestion versus extended overnight fasting increases postprandial glucose flux after exercise in healthy men. Am J Physiol. https://doi. org/10.1152/ajpendo.00163.2018
Fenn W, Rahn H, Otis A (1946) A theoretical study of the composition of the alveolar air at altitude. Am J Physiol 146:637-653

Frayn K (1983) Calculation of substrate oxidation rates in vivo from gaseous exchange. J Appl Physiol 55:628-634

Friedmann B, Bauer T, Menold E, Bartsch P (2004) Exercise with the intensity of the individual anaerobic threshold in acute hypoxia. Med Sci Sports Exerc 36(10):1737-1742. https://doi. org/10.1249/01.mss.0000142307.62181.37

Geiger P, Han D, Wright D, Holloszy J (2006) How muscle insulin sensitivity is regulated: testing of a hypothesis. Am J Physiol 291(6):E1258-E1263

Girard O, Malatesta D, Millet G (2017) Walking in hypoxia: an efficient treatment to lessen mechanical constraints and improve health in obese individuals? Front Physiol 8:73

Goodman J, Hassell K, Irwin D, Witkowski EH, Nuss R (2014) The splenic syndrome in individuals with sickle cell trait. High Alt Med Biol 15:468-471

Griffiths A, Shannon O, Matu J, King R, Deighton K, O'Hara J (2019) The effects of environmental hypoxia on substrate utilisation during exercise: a meta-analysis. J Int Soc Sports Nutr $16: 1$

Hopkins W (2004) How to interpret changes in an athletic performance test. Sportscience 8:1-7

Howley E, Bassett D, Welch H (1995) Criteria for maximal oxygen uptake: review and commentary. Med Sci Sports Exerc 27:1292-1301

Huang B, Wu P, Bowker-Kinley M, Harris R (2002) Regulation of pyruvate dehydrogenase kinase expression by peroxisome proliferator-activated receptor-alpha ligands, glucocorticoids, and insulin. Diabetes 51:276-283

Jentjens R, Moseley L, Waring R, Harding L, Jeukendrup A (2004) Oxidation of combined ingestion of glucose and fructose during exercise. J Appl Physiol 96:1277-1284

Jeukendrup A, Wallis G (2005) Measurement of substrate oxidation during exercise by means of gas exchange measurements. Int J Sports Med 26(1):28-37

Jones A, Doust J (1996) A 1\% treadmill grade most accurately reflects the energetic cost of outdoor running. J Sports Sci 14:321-327

Katayama K, Goto K, Ishida K, Ogita F (2010) Substrate utilization during exercise and recovery at moderate altitude. Metabolism 59(7):959-966. https://doi.org/10.1016/j.metabol.2009.10.017

Kayser B, Verges S (2013) Hypoxia, energy balance and obesity: from pathophysiological mechanisms to new treatment strategies. Obes Rev 14(7):579-592

König B, Rauer C, Rosenbaum S, Brandsch C, Eder K, Stangl G (1999) Fasting upregulates PPAR target genes in brain and influences pituitary hormone expression in a PPAR dependent manner. PPAR Res. https://doi.org/10.1155/2009/801609

Lundby C, Van Hall G (2002) Substrate utilization in sea level residents during exercise in acute hypoxia and after 4 weeks of acclimatization to $4100 \mathrm{~m}$. Acta Physiol Scand 176(3):195-201

Matu J, Deighton K, Ispoglou T, Duckworth L (2017) The effect of moderate versus severe simulated altitude on appetite, gut hormones, energy intake and substrate oxidation in men. Appetite 113:284-292. https://doi.org/10.1016/j.appet.2017.02.041

Matu J, Gonzalez JT, Ispoglou T, Duckworth L, Deighton K (2018) The effects of hypoxia on hunger perceptions, appetite-related hormone concentrations and energy intake: a systematic review and meta-analysis. Appetite 125:98-108. https://doi.org/10.1016/j. appet.2018.01.015

Mellor A, Bakker-Dyos J, Howard M, Boos C, Cooke M, Vincent E, Scott P, O’Hara J, Clarke S, Barlow M, Matu J, Deighton K, Hill N, Newman C, Cruttenden R, Holdsworth D, Woods D (2017) The British services Dhaulagiri medical research expedition 2016: a unique military and civilian research collaboration. J R Army Ed Corps 163(6):371-375 
Morishima T, Mori A, Sasaki H, Goto K (2014) Impact of exercise and moderate hypoxia on glycemic regulation and substrate oxidation pattern. PLoS ONE 9(10):e108629. https://doi.org/10.1371/journ al.pone. 0108629

O'Hara JP, Woods DR, Mellor A, Boos C, Gallagher L, Tsakirides C, Arjomandkhah NC, Holdsworth DA, Cooke CB, Morrison DJ, Preston T, King RF (2017) A comparison of substrate oxidation during prolonged exercise in men at terrestrial altitude and normobaric normoxia following the coingestion of $13 \mathrm{C}$ glucose and 13C fructose. Physiol Rep. https://doi.org/10.14814/phy2.13101

Péronnet F, Massicotte D, Folch N, Melin B, Koulmann N, Jimenez C, Bourdon L, Launay JC, Savourey G (2006) Substrate utilization during prolonged exercise with ingestion of $13 \mathrm{C}$-glucose in acute hypobaric hypoxia (4,300 m). Eur J Appl Physiol 97(5):527-534

Reeves S, Halsey L, McMeel Y, Huber J (2013) Breakfast habits, beliefs and measures of health and wellbeing in a nationally representative UK sample. Appetite 60:51-57

Romijn JA, Coyle E, Sidossis LS, Gastaldelli A, Horowitz JF, Endert E, Wolfe RR (1993) Regulation of endogenous fat and carbohydrate metabolism in relation to exercise intensity and duration. Am J Physiol 265(28):E380-E391

Sahlin K (1990) Muscle carnitine metabolism during incremental dynamic exercise in humans. Acta Physiol 138(3):259-262

Sergi G, Imoscopi A, Sarti S, Perissinotto E, Coin A, Inelman EM, Zambon S, Busetto L, Seresin C, Manzato E (2010) Changes in total body and limb composition and muscle strength after a 6-8 weeks sojourn at extreme altitude $(5000-8000 \mathrm{~m})$. J Sports Med Phys Fitness 50(4):450-455

Skinner JS et al (1999) Reproducibility of maximal exercise test data in the HERITAGE family study. Med Sci Sports Exerc 31:1623-1628

Spaeth AM, Dinges DF, Goel N (2015) Resting metabolic rate varies by race and by sleep duration. Obesity 23(12):2349-2356

Spriet L, Watt M (2003) Regulatory mechanisms in the interaction between $\mathrm{CHO}$ and lipid oxidation during exercise. Acta Physiol Scand 178:443-452
Stevenson E, Williams C, Nute M, Swaile P, Tsui M (2005) The effect of the glycemic index of an evening meal on the metabolic responses to a standard high glycemic index breakfast and subsequent exercise in men. Int J Sport Nutr Exerc Metab 15:308-322

Tappy L (2018) Fructose-containing caloric sweeteners as a cause of obesity and metabolic disorders. J Exp Biol 221:164202

Taylor R, Price T, Katz L, Shulman R, Shulman G (1993) Direct measurement of change in muscle glycogen concentration after a mixed meal in normal subjects. Am J Physiol 265(2):E224-E229

Tentolouris N, Tsigos C, Perea D, Koukou E, Kyriaki D, Kitsou E, Daskas S, Daifotis Z, Makrilakis K, Raptis SA, Katsilambros N (2003) Differential effects of high-fat and high-carbohydrate isoenergetic meals on cardiac autonomic nervous system activity in lean and obese women. Metabolism 52(11):1426-1432

Van Loon L, Greenhaff P, Constantin-Teodosiu D, Saris W, Wagenmakers A (2001) The effect of increasing exercise intensity on muscle fuel utilisation in humans. J Physiol 536:295-304

Watt M, Howlett K, Febbraio M, Spriet L, Hargreaves M (2001) Adrenaline increases skeletal muscle glycogenolysis, PDH activation and carbohydrate oxidation during moderate exercise in humans. J Physiol 534:269-278

Wolever T, Jenkins D, Ocana A, Rao V, Collier G (1988) Second-meal effect: low glycemic-index foods eaten at dinner improve subsequent breakfast glycemic response. Am J Clin Nutr 48:1041-1047

Young A, Evans W, Cymerman A, Pandolf K, Knapik J, Maher J (1982) Sparing effect of chronic high-altitude exposure on muscle glycogen utilization. J Appl Physiol 52(4):857-862

Publisher's Note Springer Nature remains neutral with regard to jurisdictional claims in published maps and institutional affiliations. 